\title{
Completed Loop Audit: Improving Electronic Handover of ENT Inpatients
}

Yao A, Khwaja S. Department of Otolaryngology, Stepping Hill Hospital, Stockport NHS Foundation Trust, Poplar Grove, Hazel Grove, Stockport SK2 7JE

\section{Background}

Handover is defined as the transfer accountability and responsibility for patient care. Good handover is important to:

- Maintain continuity of care

- Maintain patient safety

- Avoid errors and harm

- Avoid delayed investigations/discharge/treatment

\section{Standard}

The Royal College of Surgeons (RCS) of England Safe Handover Guidelines (March 2007) define the following as essential items to hand over:

- Name, Age, Ward, Bed Location

- Date of admission

- Consultant

- Diagnosis

- Patient clinical condition

- Relevant Ix

- Urgency/frequency of review

- Outstanding jobs

\section{Data Collection (Cycle 1)}

Data was collected retrospectively from the last 1 month from all patient electronic handover entries on the departmental shared drive.

Data was collected on whether or not the essential items indicated in the RCS guidelines where completed in the electronic handover.

\section{Analysis (Cycle 1)}

80 patient entries were included, with no exclusions.

Four items of data were particularly poorly collected: date of admission, bed location, consultant responsible, EWS patient clinical condition.

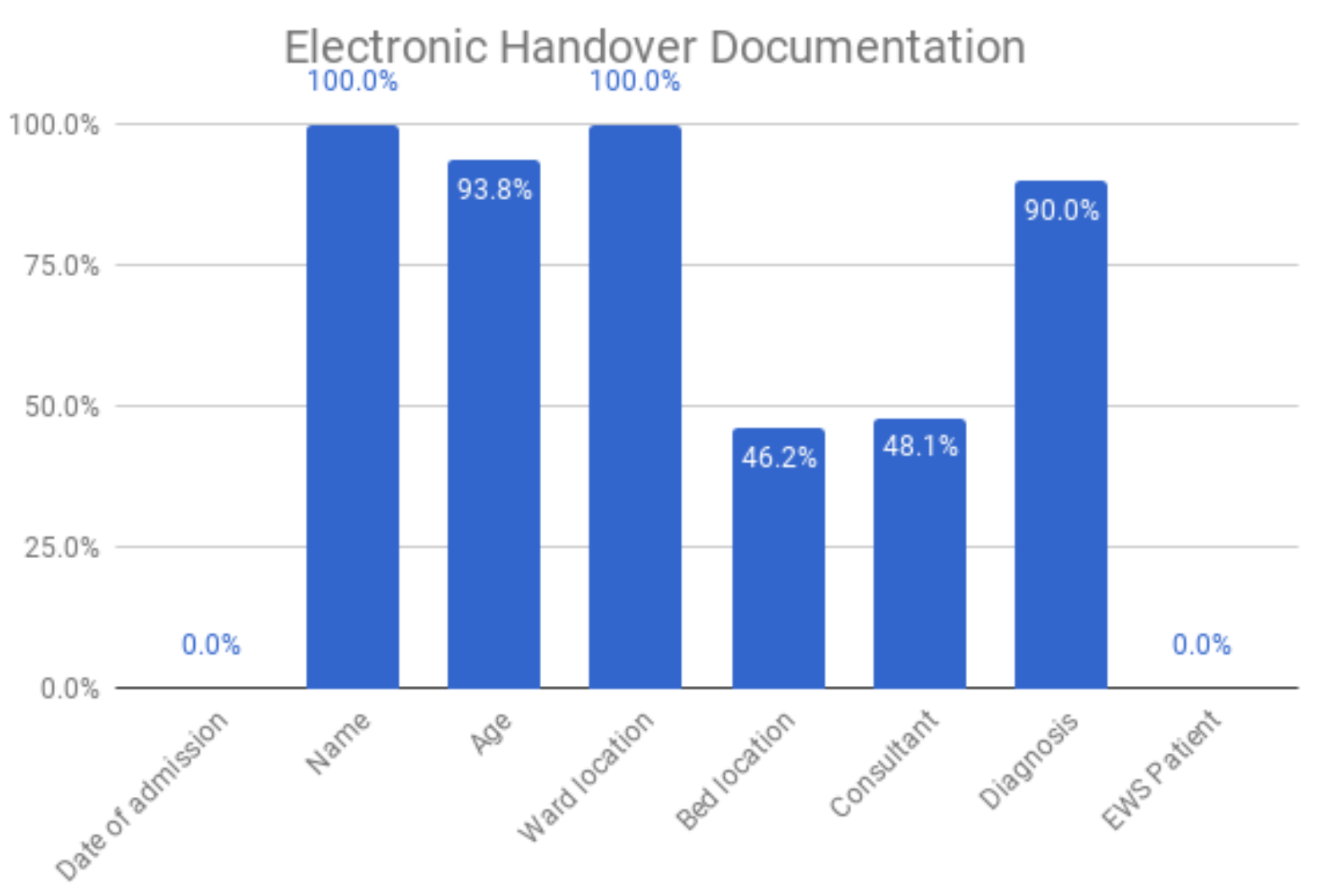

\section{Intervention: Updated Electronic Handover Tool}

We updated the existing electronic handover collection tool using Microsoft Word. Specifically we added additional and compulsory columns: date of admission, bed location, consultant responsible, and EWS/temperature/condition.

We also:

- Provided a teaching session on its use.

- Added written protocol for morning + evening handover, indicated who was responsible for updating the handover.

\section{Data Collection (Cycle 2)}

Cycle 2 data collection was performed 2.5 months later. Data was collected retrospectively using the same method as for Cycle 1. 80 patient data were collected to allow for easy comparison to the first cycle.

\section{Analysis (Cycle 2)}

80 patient entries were included.

There were significant improvements in the four items of data previously poorly collected:

- Date of admission. $0 \%$ to $91.3 \%$.

- Bed location. $46.2 \%$ to $79.7 \%$.

- Consultant responsible. $48.1 \%$ to $91.3 \%$.

- EWS patient clinical condition. $0 \%$ to $90 \%$.

We also anecdotally experienced increases in the efficiency of ward rounds.

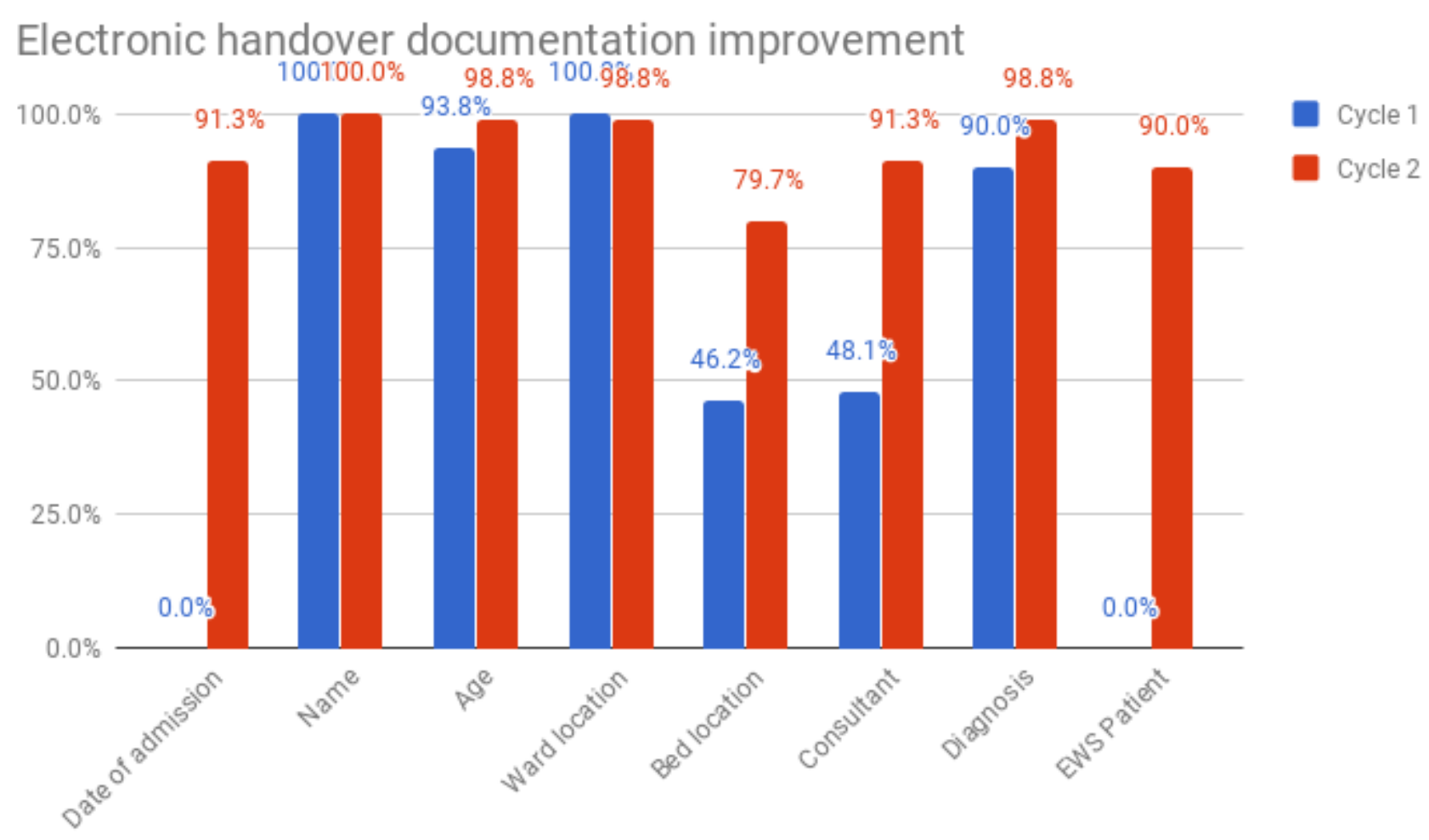

\section{Conclusions}

Simple checklist improvements can significantly improve both compliance with safe handover guidelines, but also ward round efficiency.

The use of checklists should be combined with teaching in its use, and introduction of protocols to systemise (potentially) complex information transfer. 